\title{
Decays of the vector glueball
}

\author{
Francesco Giacosa ${ }^{(a, b)} *$ Julia Sammet ${ }^{(b)}$ 用 and Stanislaus Janowski ${ }^{(b)}$ \\ (a) Institute of Physics, Jan Kochanowski University, \\ ul. Swietokrzyska 15, 25-406 Kielce, Poland, and \\ (b) Institute for Theoretical Physics, Goethe University, \\ Max-von-Laue-Str. 1, 60438 Frankfurt am Main, Germany.
}

\begin{abstract}
We calculate two- and three-body decays of the (lightest) vector glueball into (pseudo)scalar, (axial-)vector, as well as pseudovector and excited vector mesons in the framework of a model of QCD. While absolute values of widths cannot be predicted because the corresponding coupling constants are unknown, some interesting branching ratios can be evaluated by setting the mass of the yet hypothetical vector glueball to $3.8 \mathrm{GeV}$ as predicted by quenched Lattice QCD. We find that the decay mode $\omega \pi \pi$ should be one of the largest (both through the decay chain $\mathcal{O} \rightarrow b_{1} \pi \rightarrow \omega \pi \pi$ and through the direct coupling $\mathcal{O} \rightarrow \omega \pi \pi)$. Similarly, the (direct and indirect) decay into $\pi K K^{*}(892)$ is sizable. Moreover, the decays into $\rho \pi$ and $K^{*}(892) K$ are, although subleading, possible and could play a role in explaining the $\rho \pi$ puzzle of the charmonium state $\psi(2 S)$ thank to a (small) mixing with the vector glueball. The vector glueball can be directly formed at the ongoing BESIII experiment as well as at the future PANDA experiment at the FAIR facility. If the width is sufficiently small $(\lesssim 100 \mathrm{MeV})$ it should not escape future detection. It should be stressed that the employed model is based on some inputs and simplifying assumptions: the value of glueball mass (at present, the quenched lattice value is used), the lack of mixing of the glueball with other quarkonium states, and the use of few interaction terms. It then represents a first step toward the identification of the main decay channels of the vector glueball, but shall be improved when corresponding experimental candidates and/or new lattice results will be available.
\end{abstract}

PACS numbers: 12.39.Fe, 12.39.Mk, 13.20.Jf

Keywords: chiral Lagrangians, (pseudo)scalar mesons, vector glueball

\section{INTRODUCTION}

The search for glueballs is an important part of experimental as well as theoretical hadronic physics [1, 2]. Lattice QCD predicts a rich spectrum of glueball states below $5 \mathrm{GeV}[3,4]$, but up to now no predominantly glueball state could be unambiguously assigned to one of the mesons listed in the PDG [6].

The lightest glueball predicted by lattice QCD is a scalar particle. This is definitely the glueball which has been most intensively studied both experimentally and theoretically. Various theoretical approaches were developed to understand which scalar-isoscalar resonance contains the largest gluonic amount, e.g. Refs. 2, 7] and refs. therein. In most scenarios, either the resonance $f_{0}(1500)$ or the resonance $f_{0}(1710)$ has the largest gluonic content in its wave function.

The field corresponding to the scalar glueball is often related to the dilaton [8, 9]: the corresponding nonvanishing condensate is proportional to the gluon condensate and is linked to a basic feature of QCD, the trace anomaly, which allows to understand how a dimension enters into a classically dimensionless theory. Indeed, dilatation invariance and its anomalous breaking in the Yang-Mills sector have been, together with chiral symmetry, the guiding principle behind the development of an effective hadronic model of QCD, the so-called extended Linear Sigma Model (eLSM) [10, 11]. The eLSM has shown to be capable to describe various hadronic masses and decays below $1.8 \mathrm{GeV}$, as the fit in Ref. [10] confirms. The eLSM can be coupled to glueballs allowing to calculate their decays, see the following discussion. The scalar glueball is automatically present in the eLSM as a dilaton and is coupled to light mesons: its assignment and its mixing with quark-antiquark scalar fields have been studied in Ref. [11], where only one assignment was found to be acceptable: $f_{0}(1710)$ is mostly gluonic. Besides the eLSM, evidence that $f_{0}(1710)$ has a large gluonic amount is recently mounting from both lattice studies [12] and from an holographic approach [13].

The pseudoscalar glueball has also received much attention (see Ref. 14 for a review), since it is linked to another important feature of QCD: the chiral anomaly [15]. Lattice QCD predicts a mass of about $2.6 \mathrm{GeV}$, but the scenario

\footnotetext{
*Electronic address: fgiacosa@ujk.edu.pl

${ }^{\dagger}$ Electronic address: sammet@th.physik.uni-frankfurt.de

‡Electronic address: janowski@th.physik.uni-frankfurt.de
} 
in which the pseudoscalar glueball is light (at about $1.5 \mathrm{GeV}$ ) was also widely investigated, e.g. Ref. [14, 16] and refs. therein. Yet, the mismatch with the mass calculated from Lattice QCD seems to be too large for this scenario to be realistic. In a recent work, the branching ratios of a putative pseudoscalar glueball with a mass of about 2.6 $\mathrm{GeV}$ were calculated within the eLSM mentioned above [17, 18]. To this end, a chirally invariant interaction term coupling the pseudoscalar glueball to light mesons was considered. It was found that the channel $\pi \pi K$ is dominant and that the channels $\pi \pi \eta$ and $\pi \pi \eta^{\prime}$ are sizable. Such informations can be useful in future experimental search of the pseudoscalar glueball at PANDA [19] and at BESIII [20]. (Very recently, the very same interaction Lagrangian has been used to study the branching ratios of a second excited pseudoscalar glueball with a mass of about $3.7 \mathrm{GeV}$ [21].)

In this work we continue the investigation of glueballs by concentrating our attention to the vector glueball, denoted as $\mathcal{O}$. The vector glueball has received some attention in the past (e.g. in Refs. 22 26] and refs. therein, where it was studied in the context of the so-called $\rho \pi$ puzzle, see also below), but to our knowledge a systematic prediction of its decay rates has not yet been performed. Our plan is to study the vector glueball in the framework of the mentioned model of QCD, the eLSM, properly coupled to the vector glueball. As a consequence, the results are model dependent and only branching ratios can be calculated (full decay rates depend on coupling constant which cannot be calculated). Also the mass of the glueball cannot be determined within the model. We thus use the value predicted by quenched lattice QCD $(3.8 \mathrm{GeV})[3]$, well above the scalar and pseudoscalar ones [The discussion concerning the use of the lattice mass as an input and the validity of our interaction terms for the evaluation of the glueball's decays are presented in Sec. IV]. It is then expected that the vector glueball decays in two and three light mesons. We thus plan to evaluate, in the framework of our model, various branching ratios.

In order to obtain the interaction Lagrangians, we need to couple the vector glueball to conventional light mesons contained in the eLSM. Being glueballs chirally invariant fields, we assume that the interactions fulfill chiral invariance (and, at least for the dominant terms, also dilatation invariance). Moreover, in addition to the standard pseudoscalar, scalar, vector, and axial-vector mesons studied in Ref. [10], we will also consider pseudovector and excited vector mesons. It is important to stress from the very beginning that our approach could not yet be tested experimentally. (In particular, for what concerns heavy glueballs, there are at present no known candidates). Moreover, we shall also neglect mixing of the glueball with conventional quarkonium states. As we discuss more in detail later on, it will be possible to model in the future such mixing when more information will be available.

With these cautionary remarks in mind, we aim to make some observations concerning the decays of the vector glueball. In particular, we will show that, in our calculational approach, one of the most important decay modes of the vector glueball is the decay into a pseudoscalar-pseudovector pair (in particular, the channel $\mathcal{O} \rightarrow b_{1} \pi \rightarrow \omega \pi \pi$ ). These decays are obtained from an interaction term (our first effective Lagrangian for $\mathcal{O}$, denoted as $\mathcal{L}_{1}$ ) which involves the least possible number of quark-antiquark fields (only two; hence, to assure dilatation invariance, also the dilaton field is coupled). As a side effect, this analysis also offers the mathematical basis for an extension of the eLSM containing pseudovector and excited vector fields.

The second chiral Lagrangian that we build is denoted as $\mathcal{L}_{2}$ and contains the coupling of $\mathcal{O}$ to (pseudo)scalar and (axial-)vector fields. Here, three quark-antiquark fields are at first present at each vertex, hence three-body decay of $\mathcal{O}$ are automatically obtained. The decays of $\mathcal{O}$ into two pseudoscalar mesons and one vector mesons are dominant. In particular, the direct decay channels $\mathcal{O} \rightarrow \omega \pi \pi$ and $\mathcal{O} \rightarrow K^{*}(892) K \pi$ are the largest. Both the first and the second Lagrangians predict a decay into $\omega \pi \pi$ and into $K^{*}(892) K \pi$ (indirect in the first case, direct in the second), which then represent the two golden channels toward a possible future detection of the vector glueball. In the end, when condensation of scalar fields is taken into account, $\mathcal{L}_{2}$ also delivers two-body decays. Here, one vector and one scalar fields are the most relevant decay channels.

Finally, we shall also analyze a third interaction Lagrangian (denoted as $\mathcal{L}_{3}$ ), in which four quark-antiquark fields are present at each vertex. This Lagrangian breaks dilatation invariance (the coupling constant is proportional to Energy $^{-2}$ ), yet we decided to study it since it delivers interesting decays into one vector and one axial-vector pair and into one vector and one pseudoscalar pair. In particular, the $\rho \pi$ and $K^{*}(892) K$ decay modes are relevant in connection to the so-called $\rho \pi$-puzzle of the state $\psi(2 S)$, see the discussion in Sec. IV.

The vector glueball can be constructed with (at least) three constituent gluons, hence its decays and mixing are expected to be sufficiently small to allow detection (even if in the context of our model we cannot calculate them). Even if the predicted mass of $\mathcal{O}$ lies at about $3.8 \mathrm{GeV}$ and hence above the $D D$ threshold, we do not find a sizable decay into charmed mesons. Namely, the $D D$ channel turns out to be small (albeit nonzero), while other channels with a potentially sizable interaction -such as the pseudoscalar-pseudovector channel mentioned above- are not kinematically allowed when charmed mesons are considered.

In the future, the PANDA experiment at FAIR [19] will be able to form glueballs and to study their decays. In particular, the vector glueball has the quantum numbers of the photon, hence it can be also directly formed at BESIII (for which an energy scan in the region 3.5 to $4 \mathrm{GeV}$ with particular attention to the $\omega \pi \pi$ and $K^{*}(892) K \pi$ channels would be necessary). It should be mentioned that in a previous study at BES 22], a vector glueball was searched -without success- in the $\rho \pi$ channel, which is however not the favorite decay mode found in our study. 
This paper is organized as follows. In Sec. II we show the quark-antiquark nonets and their properties, while in Sec. III we introduce three effective Lagrangians and calculate decay ratios. In Sec. IV we present various discussions: the validity and the limits of the used interaction Lagrangians, the use of the mass from lattice QCD as an input, the large- $N_{c}$ limit, and the so-called $\rho \pi$ puzzle of $\psi(2 S)$. Finally, in Sec. V we outline our conclusions and outlooks. In the Appendices we show relevant details of the eLSM and of the calculations.

\section{CHIRAL MULTIPLETS}

In this Section, we concentrate on the quark-antiquark fields which represent the decay products of the vector glueball. We split our study into two parts: first, we consider (pseudo)scalar and (axial-)vector fields, which are also the basic ingredients of the eLSM when proper chiral combinations of them are taken into account. Then, in the second part we describe pseudovector mesons and excited vector mesons and the corresponding chiral combination.

\section{A. (Pseudo)scalar and (axial-)vector quark-antiquark multiplets}

The nonet of pseudoscalar fields is introduced as

$$
P=\frac{1}{\sqrt{2}}\left(\begin{array}{ccc}
\frac{\eta_{N}+\pi^{0}}{\sqrt{2}} & \pi^{+} & K^{+} \\
\pi^{-} & \frac{\eta_{N}-\pi^{0}}{\sqrt{2}} & K^{0} \\
K^{-} & K^{0} & \eta_{S}
\end{array}\right),
$$

which contains the renowned light pseudoscalar nonet $\left\{\pi, K, \eta, \eta^{\prime}\right\}[6]$, where $\eta$ and $\eta^{\prime}$ arise via the mixing $\eta=$ $\eta_{N} \cos \theta_{p}+\eta_{S} \sin \theta_{p}, \eta^{\prime}=-\eta_{N} \sin \theta_{p}+\eta_{S} \cos \theta_{p}$ with $\theta_{p} \simeq-44.6^{\circ}\left[10\right.$ ]. (Using other values as e.g. $\theta_{p}=-41.4^{\circ}$ [27] changes only slightly the results presented in this work). As a next step, we introduce the matrix of scalar fields

$$
S=\frac{1}{\sqrt{2}}\left(\begin{array}{ccc}
\frac{\sigma_{N}+a_{0}^{0}}{\sqrt{2}} & a_{0}^{+} & K_{S}^{+} \\
a_{0}^{-} & \frac{\sigma_{N}-a_{0}^{0}}{\sqrt{2}} & K_{S}^{0} \\
K_{S}^{-} & K_{S}^{0} & \sigma_{S}
\end{array}\right)
$$

that contains the fields $\left\{a_{0}(1450), K_{0}^{*}(1430), \sigma_{N}, \sigma_{S}\right\}$. (Note, the scalar quark-antiquark states lie above $1 \mathrm{GeV}[10]$, thus the nonet of light scalar states $\left\{a_{0}(980), K_{0}^{*}(800), f_{0}(500), f_{0}(980)\right\}$ is something else. A possibility is a nonet of light tetraquark states [28, 29] or a nonet of dynamically generated states [30 32]). As a first approximation, the non-strange bare field $\sigma_{N} \equiv|\bar{u} u+\bar{d} d\rangle / \sqrt{2}$ corresponds predominantly to the resonance $f_{0}(1370)$ and the bare field $\sigma_{S} \equiv|\bar{s} s\rangle$ predominantly to $f_{0}(1500)$. Finally, in the eLSM the state $f_{0}(1710)$ is predominantly a scalar glueball, see details in Ref. [11] in which the mixing matrix is presented.

Nowadays evidence toward a large gluonic amount in $f_{0}(1710)$ is increasing: besides the eLSM [11], in the recent lattice work of Ref. [12] the radiative decay $j / \psi \rightarrow \gamma G$ (where $G$ is a pure glueball) has been analyzed and found to be in good agreement with the experimental decay rate $j / \psi \rightarrow \gamma f_{0}(1710)$. Moreover, the study of Ref. [13] reaches the same conclusion in the framework of holographic QCD.

The scalar and pseudoscalar matrices are combined into

$$
\Phi=S+i P
$$

which has a simple transformation under chiral transformations $U_{L}(3) \times U_{R}(3): \Phi \rightarrow U_{L} \Phi U_{R}^{\dagger}$, where $U_{L}$ and $U_{R}$ are $U(3)$ matrices. Under parity $\Phi \rightarrow \Phi^{\dagger}$ and under charge conjugation $\Phi \rightarrow \Phi^{t}$. The matrix $\Phi$ is used as a building block in the construction of the eLSM Lagrangian, see Appendix A and Tables I and II.

We now turn to vector and axial-vector fields. The nonet of vector fields is introduced as

$$
V^{\mu}=\frac{1}{\sqrt{2}}\left(\begin{array}{ccc}
\frac{\omega_{N}+\rho^{0}}{\sqrt{2}} & \rho^{+} & K^{\star+} \\
\rho^{\mu-} & \frac{\omega_{N}-\rho^{0}}{\sqrt{2}} & K^{\star 0} \\
K^{\star-} & K^{\star 0} & \omega_{S}
\end{array}\right)^{\mu}
$$


and the nonet of axial-vector fields as

$$
A^{\mu}=\frac{1}{\sqrt{2}}\left(\begin{array}{ccc}
\frac{f_{1 N}+a_{1}^{0}}{\sqrt{2}} & a_{1}^{+} & K_{1, A}^{+} \\
a_{1}^{-} & \frac{f_{1 N}-a_{1}^{0}}{\sqrt{2}} & K_{1, A}^{0} \\
K_{1, A}^{-} & \bar{K}_{1, A}^{0} & f_{1 S}
\end{array}\right)^{\mu} .
$$

The matrix $V^{\mu}$ contains the vector states $\left\{\rho, K^{*}(892), \omega, \phi\right\}$, while the matrix $A^{\mu}$ contains the axial-vector states $\left\{a_{1}(1230), K_{1, A}, f_{1}(1285), f_{1}(1420)\right\}$, where $K_{1, A}$ is a mixture of the two physical states $K_{1}(1270)$ and $K_{1}(1400)$, see Sec. II.B. We neglect (the anyhow small) strange-nonstrange mixing, hence $\omega \equiv \omega_{N}$ and $f_{1 N} \equiv f_{1}(1285)$ are purely nonstrange mesons of the type $\sqrt{1 / 2}(\bar{u} u+\bar{d} d)$, while $\omega_{S} \equiv \phi$ and $f_{1 S} \equiv f_{1}(1285)$ are purely $\bar{s} s$ states.

Then, one defines the right-handed and left-handed combinations:

$$
R^{\mu}=V^{\mu}-A^{\mu} \text { and } L^{\mu}=V^{\mu}+A^{\mu}
$$

Under chiral transformation they transform in a compact way: $R^{\mu} \rightarrow U_{R} R^{\mu} U_{R}^{\dagger}, L^{\mu} \rightarrow U_{L} L^{\mu} U_{L}^{\dagger}$.

Details of the currents and transformations are shown in Table I and II.

The eLSM Lagrangian includes the multiplets $S, P, V$, and $A$ presented above. In addition, a dilaton/glueball field is also present in order to describe dilatation symmetry and its anomalous breaking. The details of the eLSM (together with its symmetries, most notably chiral and dilatation symmetries together with their anomalous, explicit, and spontaneous breaking terms) are briefly summarized in Appendix A and extensively presented in Refs. [10, 11] for $N_{f}=3$. (Previous versions of the eLSM for $N_{f}=2$ are discussed in Ref. 33. while an extension to $N_{f}=4$ can be found in Ref. [34]. Baryons are considered in Ref. [35], while properties at finite density are studied in Ref. [36] and at finite temperature in Ref. 37]. Recently, it was also shown that the low-energy limit of the eLSM is equivalent to chiral perturbation theory [38].)

\section{B. Pseudovector and excited vector mesons}

We aim to investigate also the decay of the vector glueball into pseudovector and excited vector mesons. To this end we introduce the matrix

$$
B^{\mu}=\frac{1}{\sqrt{2}}\left(\begin{array}{ccc}
\frac{h_{1, N}+b_{1}^{0}}{\sqrt{2}} & b_{1}^{+} & K_{1, B}^{\star+} \\
b_{1}^{-} & \frac{h_{1, N}+b_{1}^{0}}{\sqrt{2}} & K_{1, B}^{\star 0} \\
K_{1, B}^{\star-} & \bar{K}_{1, B}^{\star 0} & h_{1, S}
\end{array}\right)^{\mu}
$$

that describes the nonet of pseudovector resonances

$$
\left\{b_{1}(1230), K_{1, B}, h_{1}(1170), h_{1}(1380)\right\}
$$

Also here, the strange-nonstrange isoscalar mixing is neglected, thus $h_{1, N} \equiv h_{1}(1170)$ is a purely nonstrange state, while $h_{1, S} \equiv h_{1}(1380)$ is a purely strange-antistrange state.

The kaonic fields $K_{1, A}$ from Eq. (5) and $K_{1, B}$ from Eq. (17) mix and generate the two physical resonances $K_{1}(1270)$ and $K_{1}(1400)$ :

$$
\left(\begin{array}{l}
K_{1}^{+}(1270) \\
K_{1}^{+}(1400)
\end{array}\right)^{\mu}=\left(\begin{array}{cc}
\cos \varphi & -i \sin \varphi \\
-i \sin \varphi & \cos \varphi
\end{array}\right)\left(\begin{array}{l}
K_{1, A}^{+} \\
K_{1, B}^{+}
\end{array}\right)^{\mu}
$$

The mixing angle reads $\varphi=(56.3 \pm 4.2)^{\circ}[39]$. The same transformations hold for $K_{1}^{0}(1270)$ and $K_{1}^{0}(1400)$, while for the other kaonic states one has to take into account that $K_{1}^{-}(1270)=K_{1}^{+}(1270)^{\dagger}$ and $\bar{K}_{1}^{0}(1270)=K_{1}^{0}(1270)^{\dagger}($ and so for $\left.K_{1}^{-}(1400)\right)$.

The chiral partners of the pseudovector mesons are excited vector mesons which arise from the combination $L=2$, $S=1$ coupled to $J^{P C}=1^{--}$. The corresponding fields listed in the PDG [6] are given by

$$
\left\{\rho(1700), K^{*}(1680), \omega(1650), \phi(? ? ?)\right\}
$$


Note, $\phi(? ?$ ?) was not yet found (one expects a vector state with a mass of about $1.95 \mathrm{GeV}$ from the comparison to the radially excited vector mesons). The corresponding nonet reads:

$$
E_{\text {ang }}^{\mu}=\frac{1}{\sqrt{2}}\left(\begin{array}{ccc}
\frac{\omega_{\text {ang }, N}+\rho_{\text {ang }}^{0}}{\sqrt{2}} & \rho_{\text {ang }}^{+} & K_{\text {ang }}^{\star+} \\
\rho_{\text {ang }}^{-} & \frac{\omega_{\text {ang }, N-\rho_{\text {ang }}^{0}}}{\sqrt{2}} & K_{\text {ang }}^{\star 0} \\
K_{\text {ang }}^{\star-} & \bar{K}_{\text {ang }}^{\star 0} & \omega_{\text {ang }, S}^{\mu}
\end{array}\right)^{\mu} .
$$

We then build the matrix

$$
\tilde{\Phi}^{\mu}=E_{\text {ang }}^{\mu}-i B^{\mu}
$$

which under chiral transformations changes as $\tilde{\Phi}^{\mu} \rightarrow U_{L} \tilde{\Phi}^{\mu} U_{R}^{\dagger}$ (just as the standard (pseudo)scalar $\Phi$, hence the name), under parity as $\tilde{\Phi}^{\mu} \rightarrow \tilde{\Phi}^{\dagger \mu}$, and under charge conjugations as $\tilde{\Phi}^{\mu} \rightarrow-\tilde{\Phi}^{t, \mu}$, see Table I and II for details. Although not the goal of the present paper, one could use the matrix $\tilde{\Phi}^{\mu}$ in order to build an extension of the eLSM which includes pseudovector and excited vector mesons. This project is left for the future.

Finally, we present in the Tables I and II the summary of all relevant properties and transformations of the nonets introduced in this Section.

\begin{tabular}{|c|c|c|c|c|c|}
\hline Nonet & $L$ & $S$ & $J^{P C}$ & Current & Assignment \\
\hline$P$ & 0 & 0 & $0^{-+}$ & $P_{i j}=\frac{1}{\sqrt{2}} \bar{q}_{j} i \gamma^{5} q_{i}$ & $\pi, K, \eta, \eta^{\prime}$ \\
\hline$S$ & 1 & 1 & $0^{++}$ & $S_{i j}=\frac{1}{\sqrt{2}} \bar{q}_{j} q_{i}$ & $a_{0}(1450), K_{0}^{*}(1430), f_{0}(1370), f_{0}(1510)$ \\
\hline$V^{\mu}$ & 0 & 1 & $1^{--}$ & $V_{i j}^{\mu}=\frac{1}{\sqrt{2}} \bar{q}_{j} \gamma^{\mu} q_{i}$ & $\rho(770), K^{*}(892), \omega(785), \phi(1024)$ \\
\hline$A^{\mu}$ & 1 & 1 & $1^{++}$ & $A_{i j}^{\mu}=\frac{1}{\sqrt{2}} \bar{q}_{j} \gamma^{5} \gamma^{\mu} q_{i}$ & $a_{1}(1230), K_{1, A}, f_{1}(1285), f_{1}(1420)$ \\
\hline$B^{\mu}$ & 1 & 0 & $1^{+-}$ & $B_{i j}^{\mu}=\frac{1}{\sqrt{2}} \bar{q}_{j} \gamma^{5} \overleftrightarrow{\partial}^{\mu} q_{i}$ & $b_{1}(1230), K_{1, B}, h_{1}(1170), h_{1}(1380)$ \\
\hline$E_{\text {ang }}^{\mu}$ & 2 & 1 & $1^{--}$ & $E_{\text {ang, }, i j}^{\mu}=\frac{1}{\sqrt{2}} \bar{q}_{j} i \overleftrightarrow{\partial^{\mu}} q_{i}$ & $\rho(1700), K^{*}(1680), \omega(1650), \phi(? ? ?)$ \\
\hline
\end{tabular}

TABLE I: Summary of the quark-antiquark nonets and their properties.

\begin{tabular}{|c|c|c|c|c|}
\hline Chiral multiplet & Current & $U_{R}(3) \times U_{R}(3)$ & $P$ & $C$ \\
\hline$\Phi=S+i P$ & $\sqrt{2} \bar{q}_{R, j} q_{L, i}$ & $U_{L} \Phi U_{R}^{\dagger}$ & $\Phi^{\dagger}$ & $\Phi^{t}$ \\
\hline$R^{\mu}=V^{\mu}-A^{\mu}$ & $\sqrt{2} \bar{q}_{R, j} \gamma^{\mu} q_{R, i}$ & $U_{R} R^{\mu} U_{R}^{\dagger}$ & $L_{\mu}$ & $L^{t \mu}$ \\
\hline$L^{\mu}=V^{\mu}+A^{\mu}$ & $\sqrt{2} \bar{q}_{L, j} \gamma^{\mu} q_{L, i}$ & $U_{L} R^{\mu} U_{L}^{\dagger}$ & $R_{\mu}$ & $R^{t \mu}$ \\
\hline$\tilde{\Phi}^{\mu}=E_{\text {ang }}^{\mu}-i B^{\mu}$ & $\sqrt{2} \bar{q}_{R, j} i \stackrel{\leftrightarrow}{\partial}{ }^{\mu} q_{L, i}$ & $U_{L} \tilde{\Phi}^{\mu} U_{R}^{\dagger}$ & $\tilde{\Phi}^{\dagger \mu}$ & $-\tilde{\Phi}^{t \mu}$ \\
\hline
\end{tabular}

TABLE II: Transformation properties of the chiral multiplets.

\section{DECAY OF THE VECTOR GLUEBALL INTO CONVENTIONAL MESONS}

In this section we present the interaction terms describing the coupling of the vector glueball field $\mathcal{O}^{\mu}$ to the various quark-antiquark multiplets introduced in the previous section. Chiral symmetry as well as invariance under parity and charge conjugations are the guiding principles. In addition, dilatation invariance is assumed to hold in the two most relevant terms. A third interaction which breaks dilatation invariance and involves the Levi-Civita tensor is also considered. Branching ratios are summarized in the tables III-VI. 


\section{A. Decays into (pseudo)scalar and excited vector and pseudovector mesons}

The (nontrivial) chiral Lagrangian with the minimal number of quark-antiquark fields contains the coupling of the vector glueball to (pseudo)scalar and excited(pseudo)vector mesons:

$$
\mathcal{L}_{1}=\lambda_{\mathcal{O}, 1} G \mathcal{O}_{\mu} \operatorname{Tr}\left[\Phi^{\dagger} \tilde{\Phi}^{\mu}+\tilde{\Phi}^{\mu \dagger} \Phi\right] .
$$

We introduced also the dilaton $G$ in such a way that the interaction term has exactly dimension $4\left(\lambda_{\mathcal{O}, 1}\right.$ is dimensionless) as required by dilatation invariance (we recall that only positive or vanishing powers of $G$ are acceptable [10].) Using Table II we obtain:

$$
\mathcal{L}_{1}=\lambda_{\mathcal{O}, 1} G \mathcal{O}_{\mu} \operatorname{Tr}\left[2 S E_{\text {ang }}^{\mu}-2 P B^{\mu}\right] .
$$

Setting the dilaton field $G$ to its condensate $G=G_{0}[9]$, substituting $P \rightarrow \mathcal{P}$ as described in Appendix A, and by introducing the physical kaonic fields defined in Eqs. (9), lead to the desired expressions for the two-body decays (see Appendix B for its analytic form). In particular, we have decays of the type $\mathcal{O} \rightarrow B P$ and $\mathcal{O} \rightarrow S E_{\text {ang. The main }}$ decay channel is $\mathcal{O} \rightarrow b_{1} \pi$. We thus expect a significant decay rate of an hypothetical vector glueball into the channel

$$
\mathcal{O} \rightarrow b_{1} \pi \rightarrow \omega \pi \pi
$$

Unfortunately, we cannot determine $\lambda_{\mathcal{O}, 1}$ in the present framework, but we can easily calculate various decay ratios which represent clear predictions of the present approach, see Table III. Besides the channel $b_{1} \pi$, also the decays involving kaons and $K_{1}(1270)$ and $K_{1}(1400)$ are sizable.

In Table III (as well as in all other tables presented in this work) we keep for definiteness two significant digits for our results. It is difficult to estimate the actual uncertainty of our ratios, since various unknown factors enter. One source of error is related to the mass of the glueball, for which we used the quenched lattice result of $3.81 \mathrm{GeV}$ (one may estimate it $\sim 10-15 \%$ [3] see also the detailed discussion in Sec. IV.B and the cautionary remarks written there). Another source of uncertainty is connected to the validity of the employed effective model; indeed, in Ref. 34] an application of the eLSM to heavy charmed mesons has found to be applicable within $10 \%$ for what concerns the calculation of decays. It seems reasonable to expect a similar accuracy in the present approach, even if it is not yet possible to test the decays of heavy glueballs since they were not yet discovered. Putting altogether, we can estimate the validity of our branching ratios to about 20-30\%. However, the interesting point is that the qualitative outcomes of our study are not strongly dependent on the precise input of the parameters. For instance, in Table 1 the main information $\left(O \rightarrow b_{1} \pi\right.$ dominates $)$ is stable.

At the present state of knowledge, the decays into scalar mesons can only be approximate. For this reason, we did not 'unmix' $\sigma_{N}$ and $\sigma_{S}$. In addition, we also expect a three-body decay (when $G$ in Eq. (12) is not set to its v.e.v.):

$$
\mathcal{O} \rightarrow G b_{1} \pi
$$

Upon identifying $G$ with $f_{0}(1710)$ (the by far dominant contribution in the eLSM [11]), one obtains a very small decay ratio:

$$
\frac{\Gamma_{\mathcal{O} \rightarrow f_{0}(1710) b_{1} \pi}}{\Gamma_{\mathcal{O} \rightarrow b_{1} \pi}}=3.9 \cdot 10^{-6}
$$

There are further three-body interactions contained in $\mathcal{L}_{1}$, but they are not kinematically allowed.

Moreover, we did not include in the table the ratio $\omega_{\text {ang,S }} \equiv \phi(? ? ?)$ because the corresponding state was not yet experimentally found. Yet, assigning it to a yet hypothetical $\phi(1950)$ state, we obtain $\Gamma_{\mathcal{O} \rightarrow f_{0}(1510) \phi(1950)} / \Gamma_{\mathcal{O} \rightarrow b_{1} \pi} \simeq$ 0.037 .

We also neglect the mixing $\mathcal{O}_{\mu} \operatorname{Tr}\left[E_{\text {ang }}^{\mu}\right]$ arising when the field $S$ condenses (see Appendix A). Namely, the large mass difference between $\mathcal{O}$ and excited vector mesons assures that such mixing is negligible.

\section{B. Coupling to (pseudo)scalar and (axial-)vector mesons}

Next, we consider a chirally invariant and dilatation invariant Lagrangians which couples $\mathcal{O}$ to three quark-antiquark states. It involves both (pseudo)scalar and (axial-)vector fields:

$$
\mathcal{L}_{2}=\lambda_{\mathcal{O}, 2} \mathcal{O}_{\mu} \operatorname{Tr}\left[L^{\mu} \Phi \Phi^{\dagger}+R^{\mu} \Phi^{\dagger} \Phi\right]
$$




\begin{tabular}{|c|c|}
\hline Quantity & Value \\
\hline$\frac{\mathcal{O} \rightarrow \eta h_{1}(1170)}{\mathcal{O} \rightarrow b_{1} \pi}$ & 0.17 \\
\hline$\frac{\mathcal{O} \rightarrow \eta h_{1}(1380)}{\mathcal{O} \rightarrow b_{1} \pi}$ & 0.11 \\
\hline$\frac{\mathcal{O} \rightarrow \eta^{\prime} h_{1}(1170)}{\mathcal{O} \rightarrow b_{1} \pi}$ & 0.15 \\
\hline$\frac{\mathcal{O} \rightarrow \eta^{\prime} h_{1}(1380)}{\mathcal{O} \rightarrow b_{1} \pi}$ & 0.10 \\
\hline$\frac{\mathcal{O} \rightarrow K K_{1}(1270)}{\mathcal{O} \rightarrow b_{1} \pi}$ & 0.75 \\
\hline$\frac{\mathcal{O} \rightarrow K K_{1}(1400)}{\mathcal{O} \rightarrow b_{1} \pi}$ & 0.30 \\
\hline$\frac{\mathcal{O} \rightarrow K_{0}^{*}(1430) K^{*}(1680)}{\mathcal{O} \rightarrow b_{1} \pi}$ & 0.20 \\
\hline$\frac{\mathcal{O} \rightarrow a_{0}(1450) \rho(1700)}{\mathcal{O} \rightarrow b_{1} \pi}$ & 0.14 \\
\hline$\frac{\mathcal{O} \rightarrow f_{0}(1370) \omega(1650)}{\mathcal{O} \rightarrow b_{1} \pi}$ & 0.034 \\
\hline
\end{tabular}

TABLE III: Branching ratios for the decay of the vector glueball into a psuedoscalar-pseudovector pair and into a scalar-excitedvector pair.

where $\lambda_{\mathcal{O}, 2}$ is a dimensionless (unknown) coupling constant. By taking into account the expression listed in Table II, one obtains terms delivering three-body and two-body decays. The three-body decays are given by:

$$
\mathcal{L}_{2, \text { three-body }}=\lambda_{\mathcal{O}, 2} \mathcal{O}_{\mu} \operatorname{Tr}\left[2 V^{\mu}\left(P^{2}+S^{2}\right)\right]+2 \lambda_{\mathcal{O}, 2} \mathcal{O}_{\mu} \operatorname{Tr}\left[A^{\mu} 2 i[P, S]\right]
$$

hence decays of the type $\mathcal{O} \rightarrow V P P, \mathcal{O} \rightarrow V S S, \mathcal{O} \rightarrow A P S$, and $\mathcal{O} \rightarrow P P S$ (the later obtained by the shift $A \rightarrow Z w \partial P$, see details in Appendix B) follow. One of the most relevant decay (the second in magnitude) is

$$
\mathcal{O} \rightarrow \omega \pi \pi,
$$

which we use as reference for the ratios listed in Table IV (see Appendix $\mathrm{C}$ for the analytic expression). The channel $\mathcal{O} \rightarrow \pi K K^{*}(892)$ turns out to be the largest, followed by $\omega \pi \pi$. In the last line of Table IV we have also reported, as an example, a three-body decay into $a_{0}(1450) a_{0}(1450) \omega$, which is however very small. This is the case for all kinematically allowed $V S S$ decays.

Quite interestingly, the most prominent decay of the Lagrangian $\mathcal{L}_{1}$ is $\mathcal{O} \rightarrow b_{1} \pi \rightarrow \omega \pi \pi$ (see Table III), hence it also generates a $\omega \pi \pi$ final state (the state $b_{1}$ has a dominant decay into $\omega \pi$ ). At a first approximation, one can write

$$
\Gamma_{\mathcal{O} \rightarrow \omega \pi \pi}^{\text {tot }} \simeq \Gamma_{\mathcal{O} \rightarrow \omega \pi \pi}^{\text {direct-from }} \mathcal{L}_{2}+\Gamma_{\mathcal{O} \rightarrow b_{1} \pi \rightarrow \omega \pi \pi}^{\text {indirect-from } \mathcal{L}_{1}}
$$

although strictly speaking interferences can appear (usually they are smaller than $10 \%$, see the discussion in Ref. [18]; we will neglect such interferences in the following). Anyway, both $\mathcal{L}_{1}$ and $\mathcal{L}_{2}$ agree in predicting a strong signal into the final state $\omega \pi \pi$. Similarly, the final state into $\pi K K^{*}(892)$ is also relevant, since it comes directly from $\mathcal{L}_{2}$ and indirectly from $\mathcal{L}_{1}$ via the channels $K K_{1}(1270) \rightarrow K \pi K^{*}(892)$ and $K K_{1}(1400) \rightarrow K \pi K^{*}(892)$ (but the decays of $K_{1}(1270)$ and $K_{1}(1400)$ do not have a single dominating channel [6]).

Two-body decays from $\mathcal{L}_{2}$ are obtained when one of the $\Phi$ condenses, $\Phi \rightarrow \Phi_{0}+\Phi$ :

$$
\mathcal{L}_{2, \text { two-body }}=\lambda_{\mathcal{O}, 2} \mathcal{O}_{\mu} \operatorname{Tr}\left[V^{\mu} 2\left\{\Phi_{0}, S\right)\right]-\lambda_{\mathcal{O}, 2} \mathcal{O}_{\mu} \operatorname{Tr}\left[A^{\mu} 2 i\left[\Phi_{0}, P\right]\right]
$$

Then, the decays $\mathcal{O} \rightarrow V S, \mathcal{O} \rightarrow A P$, and $\mathcal{O} \rightarrow P P$ follow (expressions in Appendix B). The (most relevant) decay ratios are listed in Table V. The second term in Eq. (20) is suppressed because the decay amplitudes are proportional to the chirally suppressed difference $\left(\phi_{N}-\sqrt{2} \phi_{S}\right)$ (this quantity vanishes in the $U_{V}(3)$ limit). Hence both decay channels $\mathcal{O} \rightarrow A P$ and $\mathcal{O} \rightarrow P P$ are expected to be very small. In particular, $\mathcal{L}_{2, \text { two-body }}$ contains only interaction 


\begin{tabular}{|c|c|}
\hline Quantity & Value \\
\hline$\frac{\mathcal{O} \rightarrow K K \rho}{\mathcal{O} \rightarrow \omega \pi \pi}$ & 0.50 \\
\hline$\frac{\mathcal{O} \rightarrow K K \omega}{\mathcal{O} \rightarrow \omega \pi \pi}$ & 0.17 \\
\hline$\frac{\mathcal{O} \rightarrow K K \phi}{\mathcal{O} \rightarrow \omega \pi \pi}$ & 0.21 \\
\hline$\frac{\mathcal{O} \rightarrow \pi K K^{*}(892)}{\mathcal{O} \rightarrow \omega \pi \pi}$ & 1.2 \\
\hline$\frac{\mathcal{O} \rightarrow \eta \eta \omega}{\mathcal{O} \rightarrow \omega \pi \pi}$ & 0.064 \\
\hline$\frac{\mathcal{O} \rightarrow \eta \eta^{\prime} \omega}{\mathcal{O} \rightarrow \omega \pi \pi}$ & 0.019 \\
\hline$\frac{\mathcal{O} \rightarrow \eta^{\prime} \eta^{\prime} \omega}{\mathcal{O} \rightarrow \omega \pi \pi}$ & 0.019 \\
\hline$\frac{\mathcal{O} \rightarrow \eta \eta \phi}{\mathcal{O} \rightarrow \omega \pi \pi}$ & 0.039 \\
\hline$\frac{\mathcal{O} \rightarrow \eta \eta^{\prime} \phi}{\mathcal{O} \rightarrow \omega \pi \pi}$ & 0.011 \\
\hline$\frac{\mathcal{O} \rightarrow \eta^{\prime} \eta^{\prime} \phi}{\mathcal{O} \rightarrow \omega \pi \pi}$ & 0.011 \\
\hline$\frac{\mathcal{O} \rightarrow a_{0}(1450) a_{0}(1450) \omega}{\mathcal{O} \rightarrow \omega \pi \pi}$ & 0.00029 \\
\hline
\end{tabular}

TABLE IV: Branching ratios for the direct three-body decay of the vector glueball into two (pseudo)scalar and one vector meson.

\begin{tabular}{|c|c|}
\hline Quantity & Value \\
\hline$\frac{\mathcal{O} \rightarrow a_{0}(1450) \rho}{\mathcal{O} \rightarrow \omega \pi \pi}$ & 0.47 \\
\hline$\frac{\mathcal{O} \rightarrow f_{0}(1370) \omega}{\mathcal{O} \rightarrow \omega \pi \pi}$ & 0.15 \\
\hline$\frac{\mathcal{O} \rightarrow K_{0}^{*}(1430) K^{*}(892)}{\mathcal{O} \rightarrow \omega \pi \pi}$ & 0.30 \\
\hline$\frac{\mathcal{O} \rightarrow K K}{\mathcal{O} \rightarrow \omega \pi \pi}$ & 0.018 \\
\hline
\end{tabular}

TABLE V: Branching ratios for the two-body decay of the vector glueball into one (pseudo)scalar and one (axial-)vector mesons and into a kaon-kaon pair.

terms of the vector glueball with $K_{1, A} K$. Due to the fact that the $K_{1, A}$ is a mixture of $K_{1}(1270)$ and $K_{1}(1400)$ (Eq. (91)), one would obtain decay rates into $K_{1}(1270) K$ and $K_{1}(1400) K$, which are already included in Table III. Strictly speaking, one could describe these decays only once the ratio $\lambda_{\mathcal{O}, 2} / \lambda_{\mathcal{O}, 1}$ is known. However, the contribution proportional to $\lambda_{\mathcal{O}, 2}$ is suppressed, hence it represents a correction to the results listed in table III. These decay modes are then omitted from Table V. The decay into two kaons (also proportional to $\left(\phi_{N}-\sqrt{2} \phi_{S}\right)$ ) is small as expected.

Finally, the decay into $D D$ (the only decay in charmed mesons which is kinematically allowed) can be obtained by using the extension of the eLSM to the four-flavor case [34]. Due to the fact that chiral symmetry can be only considered as very approximate when charmed mesons are considered, the prediction offers only a qualitative result. 
Anyway, the ratio

$$
\frac{\Gamma_{\mathcal{O} \rightarrow D D}}{\Gamma_{\mathcal{O} \rightarrow \omega \pi \pi}} \approx 0.029
$$

shows that the $\bar{D} D$ mode is also expected to be small. This result is important because it shows that the vector glueball, even if according to lattice QCD has a mass above the $\bar{D} D$ threshold, decays only rarely in charmed mesons. The (direct and indirect) $\omega \pi \pi$ and $\pi K K^{*}(892)$ decay modes are expected to by much larger.

\section{Coupling to (axial-) vector mesons}

\begin{tabular}{|c|c|}
\hline Quantity & Value \\
\hline$\frac{\mathcal{O} \rightarrow K K^{*}(892)}{\mathcal{O} \rightarrow \rho \pi}$ & 1.3 \\
\hline$\frac{\mathcal{O} \rightarrow \eta \omega}{\mathcal{O} \rightarrow \rho \pi}$ & 0.16 \\
\hline$\frac{\mathcal{O} \rightarrow \eta^{\prime} \omega}{\mathcal{O} \rightarrow \rho \pi}$ & 0.13 \\
\hline$\frac{\mathcal{O} \rightarrow \eta \phi}{\mathcal{O} \rightarrow \rho \pi}$ & 0.21 \\
\hline$\frac{\mathcal{O} \rightarrow \eta^{\prime} \phi}{\mathcal{O} \rightarrow \rho \pi}$ & 0.18 \\
\hline$\frac{\mathcal{O} \rightarrow \rho a_{1}(1230)}{\mathcal{O} \rightarrow \rho \pi}$ & 1.8 \\
\hline$\frac{\mathcal{O} \rightarrow \omega f_{1}(1285)}{\mathcal{O} \rightarrow \rho \pi}$ & 0.55 \\
\hline$\frac{\mathcal{O} \rightarrow \omega f_{1}(1420)}{\mathcal{O} \rightarrow \rho \pi}$ & 0.82 \\
\hline
\end{tabular}

TABLE VI: Branching ratios for the decay of the vector glueball into a vector-pseudoscalar and vector-axial-vector pair.

As a last interaction term we consider an expression which breaks dilatation invariance:

$$
\mathcal{L}_{3}=\alpha \varepsilon_{\mu \nu \rho \sigma} \partial^{\rho} \mathcal{O}^{\sigma} \operatorname{Tr}\left[L^{\mu} \Phi R^{\nu} \Phi^{\dagger}\right]
$$

where $\alpha$ has dimension of energy ${ }^{-2}$. Even if it is expected to lead to smaller decay rates than the previous two Lagrangians, the presence of an 'anomalous' Levi-Civita tensor may point to a non-negligible interaction strength even in presence of an explicit breaking of dilatation symmetry.

We restrict our study to the case in which the scalar fields condenses. Hence, upon setting $\Phi=\Phi_{0}$, we obtain:

$$
\mathcal{L}_{3, \text { two-body }}=2 \alpha \varepsilon_{\mu \nu \rho \sigma} \partial^{\rho} \mathcal{O}^{\sigma} \operatorname{Tr}\left[A^{\mu} \Phi_{0} V^{\nu} \Phi_{0}\right]
$$

which leads to $O \rightarrow A V$ and $O \rightarrow P V$ (upon shifting $A$ ). We chose the decay channel $\mathcal{O} \rightarrow \rho \pi$ as our reference decay mode (see Appendix B for its expression). In Table VI we present the branching ratios which follow from $\mathcal{L}_{3}$. The dominant decay modes are and $\rho \pi, K K^{*}(892)$, and $\rho a_{1}(1230)$ (with increasing strength). The $\rho \pi$ and $K K^{*}(892)$ modes are also important in the description of the $\rho \pi$ puzzle of $\psi(2 S)$ described in the next Section.

\section{DISCUSSIONS}

In this section we discuss in detail some important issues related to our approach and our results. First, we motivate the applicability of our interaction Lagrangians, second we justify the use of glueball's masses form lattice QCD as an input, and third we present the so-called $\rho \pi$-puzzle and its connection to the vector glueball. 


\section{A. On the applicability of the interaction Lagrangians}

The eLSM is a low-energy chiral model valid up to $1.7 \mathrm{GeV}$, while the vector glueball studied in this work has a mass of about $3.8 \mathrm{GeV}$. It should be stressed that the joint model

$$
\mathcal{L}_{\text {eLSM }}+\mathcal{L}_{O-\mathrm{eLSM}}
$$

(where $\mathcal{L}_{O \text {-eLSM }}=\mathcal{L}_{1}+\mathcal{L}_{2}+\mathcal{L}_{2}$ is the sum of the three interaction terms described in the previous section) should be regarded as a model suited to calculate exclusively the decays of the field $\mathcal{O}$. However, one should not use such a model to calculate, for instance, pion-pion scattering or analogous quantities up to the energy of about $4 \mathrm{GeV}$, since the approach is clearly not complete for that purpose.

Approaches which couple one heavy field to light mesons were widely used in the literature, e.g. Refs. [16, 42, 43] and refs. therein. The idea behind these approaches can be explained at best with a simple example: the decay of the scalar charmonium state $\chi_{c 0}$ with a mass of $3.41 \mathrm{GeV}$ into two light pseudoscalar mesons. The field $\chi_{c 0}$ is flavor-blind and, by requiring that the whole interaction Lagrangian is invariant under $U(3)_{V}$ flavor transformations, one is led to the toy-model Lagrangian

$$
\mathcal{L}_{\text {toy }}=g_{\chi} \chi_{c 0} \operatorname{Tr}\left[P^{t} P\right]
$$

where $P$ is the $3 \times 3$ matrix of pseudoscalar mesons (see Sec. II) and $g_{\chi}$ is an effective coupling constant. Upon expanding, one obtains $\mathcal{L}_{\text {toy }}=g_{\chi} \chi_{c 0}\left(\vec{\pi}^{2}+2 K^{+} K^{-}+2 K^{0} \bar{K}^{0}+\eta^{2}+\eta^{\prime 2}\right)$. By taking into account phase space, the theoretical decay ratios read $\Gamma_{\chi_{c 0 \rightarrow K K}} / \Gamma_{\chi_{c 0 \rightarrow \pi \pi}}=1.28, \Gamma_{\chi_{c 0 \rightarrow \eta \eta}} / \Gamma_{\chi_{c 0 \rightarrow \pi \pi}}=0.32$, and $\Gamma_{\chi_{c 0 \rightarrow \eta^{\prime} \eta^{\prime}}} / \Gamma_{\chi_{c 0 \rightarrow \pi \pi}}=0.28$, which should be compared to the experimental results of $1.42 \pm 0.14,0.35 \pm 0.04$, and $0.23 \pm 0.04$, respectively [6] . Considering that this toy approach is very simple and does not contain any violation of flavor symmetry (it involves only the dominant term in the large- $N_{c}$ expansion, see below), it works remarkably well when compared to data. Quite interestingly, the toy model also predicts that $\Gamma_{\chi_{c 0 \rightarrow \pi^{0} \eta}}=\Gamma_{\chi_{c 0 \rightarrow \pi^{0} \eta^{\prime}}}=\Gamma_{\chi_{c 0 \rightarrow \eta \eta^{\prime}}}=0$ (the first two results are a consequence of isospin symmetry, naturally included in $U(3)_{V}$ flavour symmetry). Indeed, none of these decays has been seen in the experiment and only stringent upper values exist [6].

The discussed toy model for the resonance $\chi_{c 0}$ is clearly limited, but shows an important fact: the decays of an heavy state (such as a charmonium resonance) into light mesons still respects the underlying symmetries of the light system (which is simply flavor symmetry in the present example). This is the case even if the employed toy-model cannot be considered as a full hadronic model valid up to the mass of charm-anticharm states. Indeed, this principle has been utilized in a large number of papers, see for example Ref. [16, 42] for what concerns the decay of the heavy charmonium state $j / \psi$ into pseudoscalar mesons and Ref. [43] for what concerns decays of $j / \psi$ involving scalar mesons.

In the present work, the calculation of the decays of the vector glueball follows the very same simple idea. Indeed, in decays of charmonia, one has first a conversion to gluons, which then transform to light mesons. Intuitively speaking, the glueball is dominated by gluons, a situation which is similar to the intermediate state of a charmonium decay. Moreover, glueballs have some features similar to that of ordinary mesons: they are made of heavy constituent gluons [44] and their size does not seem to be different from that of ordinary quark-antiquark states [45]. Hence, considerations based on symmetry appear to be a valid starting point to get some informations on decays ratios such as the ones of the vector glueball presented in this work. Clearly, only the future experimental discovery of glueballs and/or advanced lattice calculations (see the next subsection) will be able to test this basic assumption of effective hadronic models.

In order to be more realistic, in the present work a better and more complete low-energy model with (pseudo)scalar and (axial-)vector states was used: the eLSM. As explained previously, this hadronic model is based on chiral symmetry and dilatation invariance (as well as explicit, anomalous, and spontaneous breaking of these symmetries), it contains a finite number of terms, and has been used to describe meson phenomenology up to $1.7 \mathrm{GeV}$. (It is interesting to note that in Ref. [34], the eLSM was also applied to charmed mesons. It was found that the decays of such heavy mesons still approximately reflect chiral symmetry.) Moreover, we took into account that the heavy field that we couple to the eLSM, the vector glueball, is not only flavour blind, but also chirally blind: in this way chiral symmetry (together with its spontaneous breaking) has an influence on the determination of the decays. While a direct comparison with data is not yet possible, predictions can be obtained by the calculation of decay ratios. These predictions are model dependent and still neglect symmetry braking terms and mixing effects. Yet, some branching ratios might be useful in future search of the vector glueball. Moreover, the same approach can be actually applied to all glueballs listed in the lattice spectrum of Ref. [3], as the example of the vector glueball or the example of the pseudoscalar glueball show [17]. 


\section{B. On the glueball masses}

At present, the best theoretical method to calculate glueball's masses is lattice, since it numerically simulates the Yang-Mills (or the QCD) Lagrangian, hence it takes into account the nonperturbative nature of interactions involving gluons. The masses calculated in Ref. [3] (whose results are also reported in the 'quark model' summary of the PDG [6]) were obtained in the so-called quenched approximation (no quark fluctuations). However, in Ref. [5] an unquenched calculation has been performed. In the conclusions of Ref. [5] it is written: "The most conservative interpretation of our results is that the masses in terms of lattice representations are broadly consistent with results from quenched QCD." This is indeed a promising result for model builders. If mass shifts due to unquenching are not too large, one may be -with due care- optimistic that quark-antiquark admixtures do not spoil the presented picture. On the other hand, it must be stressed that the study of Ref. [5] has not been repeated yet by other groups [see however the very recent two-flavour study of light glueballs in Ref. [46], where the masses also do not vary much when quarks are included]. A full study involving glueballs and their mixing with conventional mesons would be very useful to advance in the field. This is unfortunately not an easy task. As stated in Refs. [47, 48], glueballs on the Lattice become challenging in full QCD and it is very hard to determine their admixture in physical resonances. Namely, glueball signals deteriorate fast into noise, making them very hard to extract.

In connection to hadronic models effective models, it should be however also stressed that the masses of glueballs which enter the Lagrangian should be the quenched ones (and not the unquenched). Namely the unquenching can be performed within the hadronic model. This is the case of the scalar glueball studied in Ref. [11]. We also recall that lattice is the best among many different approaches toward the calculations of the spectrum of glueballs, see Refs. [1, 49] for a list of various results. Even in the original works based on bag models, e.g. Ref. [50], the vector glueball turned out to be quite heavy (about 3-4 GeV). Quite interestingly, AdS/QCD also finds a mass of the vector state within the same range [51]. If, as we shall describe below, the width of the vector glueball turns out to be sufficiently narrow (surely, the ratio $\Gamma /\left(M-E_{\text {threshold }}\right)$ is expected to be sufficiently smaller than 1$)$, shifts due to mesonic quantum fluctuations [52] are also suppressed and shall not change drastically the mass.

Yet, in order to study mixing effects within hadronic models, first one needs to identify some promising candidates. Namely, the mixing is strongly dependent on the mass difference between nearby bare states. Its study will be possible (and highly needed) when more information will be available. As we shall discuss in the next subsection, at least in one case such mixing was estimated to be rather small.

Summarizing, it is important to say that a change in the mass of the vector glueball of about $300 \mathrm{MeV}$ (above or below the value $3.8 \mathrm{GeV}$ used in this paper) shall not change the overall picture. At present, the use of the well established quenched lattice value of Ref. [3] for the vector glueball seems the most reasonable choice to start with.

\section{On the $\rho \pi$ puzzle}

The decay of the vector glueball $\mathcal{O} \rightarrow \rho \pi$ and $\mathcal{O} \rightarrow K K^{*}(892)$ (which arise from $\mathcal{L}_{3}$, see Table VI) are interesting in connection to the so-called $\rho \pi$ puzzle [23, 24, 26]. This puzzle has to do with the experimentally missing $\rho \pi$ and $K K^{*}(892)$ decays of the resonance $\psi(2 S)$. This state is (predominately) a charmonium which emerges as a radial excitation of the famous $j / \psi$ meson. Its mass is $3.6806109 \mathrm{GeV}$ (quite close to the mass of the vector glueball evaluated by lattice QCD) and its decay width is very small: $\Gamma_{\psi(2 S)}=0.298 \mathrm{MeV}$. Due to the similarity of $j / \psi$ and $\psi(2 S)$, one expects that the ratio

$$
\frac{\Gamma_{\psi(2 S) \rightarrow \text { a certain light meson channel }}}{\Gamma_{j / \psi \rightarrow \text { a certain light meson channel }}} \simeq 0.14,
$$

holds for all light channels. The value 0.14 (the $14 \%$ rule) is the ratio of the decays into $e^{+} e^{-}[22$ ]. This rule works pretty well for various decay channels, but is badly broken for $\rho \pi$ and $K K^{*}(892)$ channels, which are clearly seen for $j / \psi$ but, as mentioned, are not seen for $\psi(2 S)$.

A possibility to solve this puzzle invokes the presence of a nearby vector glueball. A mixing of a bare charmonium $\bar{c} c$ state and a bare glueball $\mathcal{O} \equiv g g g$ leads to two physical states:

$$
\left(\begin{array}{c}
\psi(2 S) \\
\mathcal{O}^{\prime}
\end{array}\right)=\left(\begin{array}{cc}
\cos \theta & \sin \theta \\
-\sin \theta & \cos \theta
\end{array}\right)\left(\begin{array}{c}
\bar{c} c(\text { with } n=2, L=0, S=1) \\
\mathcal{O} \equiv g g g
\end{array}\right) .
$$

Then, within this picture the state $\psi(2 S)$ does not correspond to a pure charmonium, but contains (a small) glueball amount. In Ref. [24] it is estimated that $|\theta| \lesssim 2^{\circ}$. This is in agreement with the fact that such a glueball-quarkonium mixing is suppressed in the large- $N_{c}$ limit and by the fact that the vector glueball contains (at least) three constituents gluons. 


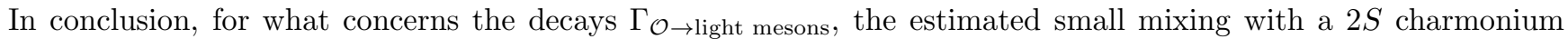
state, has a small influence, thus justifying a posteriori the results presented in this work. However, a precise study of this small mixing must be left for the future (when and if a vector glueball candidate will be found in that mass region).

\section{CONCLUSIONS AND OUTLOOK}

In this work we have presented three chirally invariant effective interaction terms describing two-body and threebody decays of a not-yet discovered vector glueball into (pseudo)scalar, (axial-)vector and pseudo(excited-)vector mesons. While the intensity of the coupling constant cannot be determined, one can predict, in the context of our model, some decay ratios and thus determine which decay channels are expected to dominate. Hopefully, our results, even if model dependent and subject to various uncertainties (validity of the symmetries used to write the Lagrangians, the value of input's mass of the vector glueball, and the absence of mixing, see below), can be of some help in future experimental search of the vector glueball. In particular, we have found the following outcomes. In the first two interaction terms (which are also dilatation invariant and are expected to be dominant) the main decay channels are $\mathcal{O} \rightarrow b_{1} \pi \rightarrow \omega \pi \pi$ (first term) as well as $\mathcal{O} \rightarrow \omega \pi \pi$ and $\mathcal{O} \rightarrow \pi K K^{*}(892)$ (second term), see Table III, IV, and V for all results. Interestingly, the first and second terms predict sizable $\omega \pi \pi$ and $\pi K K^{*}(892)$ final states, which according to our results represent the golden channels for the identification of a vector glueball's candidate. Our third interaction terms breaks dilatation invariance but was considered because it predicts decays into one vector and one pseudoscalar meson, in particular $\mathcal{O} \rightarrow \rho \pi$ and $\mathcal{O} \rightarrow K K^{*}(892)$ (Table VI). In turn, these channel may help to understand the $\rho \pi$ puzzle of $\psi(2 S)$.

The width of the vector glueball (and of glueballs in general) cannot yet be determined theoretically. According

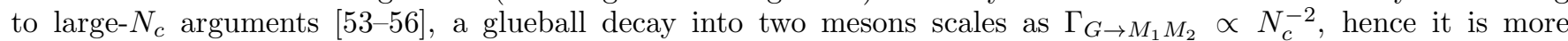
suppressed than OZI-allowed decays of conventional mesons (such as $\rho \rightarrow \pi \pi$ ) which scale $\Gamma_{M \rightarrow M_{1} M_{2}}^{\mathrm{OZI} \text {-allowed }} \propto N_{c}^{-1}$, but less suppressed than OZI-forbidden decays (such as $j / \psi \rightarrow$ light mesons [57, 58]), which scale $\Gamma_{M \rightarrow M_{1} M_{2}}^{\mathrm{OZI} \text { ] } M_{2}} \propto N_{c}^{-3}$. Large- $N_{c}$ considerations represent only a qualitative statement (and could be well broken for the physical value $N_{c}=3$, as it is e.g. in the case of the axial anomaly), but they support the hope that (at least some) glueballs are not too wide, hence one can be cautiously optimistic that (some) glueballs can be detected in the future (in particular, at the PANDA experiment [19], designed also for that purpose). Moreover, the vector glueball studied in this work is built out of (at least) three constituent gluons, for which decay requires also the complete annihilations of three gluons, hence possibly not too large.

For what concerns the mass of the glueball, we have used $3.81 \mathrm{GeV}$ as determined in Ref. [3]. Even if the uncertainty is still large, the picture concerning the main decay channels does not qualitatively change when varying the input's mass. In addition, unquenching effects do not seem to completely change the picture of glueballs [5], but future lattice studies are needed to confirm this result and to quantify deviations. In this respect, the coupling of glueballs to mesons can be a very interesting future achievement of lattice QCD. Namely, it will be possible to further constraint models such as the one described in this work.

The mixing of the glueball with other nearby quarkonium states needs also to be studied in the future. In one particular case, the mixing of the vector glueball with the predominantly charmonium state $\psi(2 S)$ has been estimated to be rather small 24]. At present, the lack of candidates for the vector glueball make a study of mixing not yet possible (in fact, mixing strongly depends on the precise value of the masses, which are not yet known). In this work, as a first, necessary step, we thus aimed to study the main interaction terms of a bare, unmixed vector glueball. As soon as candidates will be found, it will be very interesting and exciting to study mixing in more detail.

All the remarks above shows that there is a lot of room for improvement of our approach in the future. New lattice results and experimental findings will be of great help to advance in this difficult but exciting field of QCD. In this respect, this work represents a first step toward the search of the vector glueball, whose main goal is the identification of the possible dominating decay channels.

Other glueball states can be studied by following the same procedure outlined in this work. For instance, the tensor glueball $\left(J^{P C}=2^{++}\right)$is expected to have a mass of about $2.2 \mathrm{GeV}$ (it is the second lightest glueball according to lattice QCD [3]); a good candidate could be the very narrow resonance $f_{J}(2220)$ [40, 41] (at present the options are $J=2$ or 4 [6]; further experimental information is needed). A future study within the eLSM should include -besides the states included in this work- also tensor mesons and their chiral partners, the pseudotensor mesons. In addition to the tensor glueball, one has a full tower of states listed by lattice QCD: pseudotensor glueball, pseudovector glueball, oddballs (glueball with exotic quantum numbers such as $J^{P C}=0^{+-}$and $J^{P C}=2^{+-}$) as well as various glueballs with $J=3$. Various branching ratios are parameter-free once the mass of the glueball is fixed and offer a useful information toward the future search of these important (and still missing) states of QCD.

As a final remark, it must be stressed that the upcoming PANDA experiment [19] is tailor-made for the search 
of glueballs, since almost all glueballs (with the exceptions of oddballs) can be directly formed in proton-antiproton fusion processes.

\section{Acknowledgments}

The authors thank Dirk H. Rischke for useful discussions. F.G. acknowledges support from the Polish National Science Centre NCN through the OPUS project nr. 2015/17/B/ST2/01625. S.J. acknowledges support from H-QM and HGS-HIRe.

\section{Appendix A: The eLSM}

The Lagrangian of the eLSM is built by requiring chiral symmetry $\left(U(3)_{R} \times U(3)_{L}\right)$, dilatation invariance, as well as invariances under charge conjugation $C$ and parity $P$ :

$$
\begin{aligned}
\mathcal{L}_{\text {mes }} & =\mathcal{L}_{\text {dil }}+\operatorname{Tr}\left[\left(D_{\mu} \Phi\right)^{\dagger}\left(D^{\mu} \Phi\right)\right]-m_{0}^{2}\left(\frac{G}{G_{0}}\right)^{2} \operatorname{Tr}\left(\Phi^{\dagger} \Phi\right)-\lambda_{1}\left[\operatorname{Tr}\left(\Phi^{\dagger} \Phi\right)\right]^{2}-\lambda_{2} \operatorname{Tr}\left(\Phi^{\dagger} \Phi\right)^{2} \\
& -\frac{1}{4} \operatorname{Tr}\left[\left(L^{\mu \nu}\right)^{2}+\left(R^{\mu \nu}\right)^{2}\right]+\operatorname{Tr}\left[\left(\frac{m_{1}^{2}}{2}\left(\frac{G}{G_{0}}\right)^{2}+\Delta\right)\left(L_{\mu}^{2}+R_{\mu}^{2}\right)\right]+\operatorname{Tr}\left[H\left(\Phi+\Phi^{\dagger}\right)\right] \\
& +c_{1}\left(\operatorname{det} \Phi-\operatorname{det} \Phi^{\dagger}\right)^{2}+i \frac{g_{2}}{2}\left\{\operatorname{Tr}\left(L_{\mu \nu}\left[L^{\mu}, L^{\nu}\right]\right)+\operatorname{Tr}\left(R_{\mu \nu}\left[R^{\mu}, R^{\nu}\right]\right)\right\} \\
& +\frac{h_{1}}{2} \operatorname{Tr}\left(\Phi^{\dagger} \Phi\right) \operatorname{Tr}\left(L_{\mu}^{2}+R_{\mu}^{2}\right)+h_{2} \operatorname{Tr}\left[\left|L_{\mu} \Phi\right|^{2}+\left|\Phi R_{\mu}\right|^{2}\right] \\
& +2 h_{3} \operatorname{Tr}\left(L_{\mu} \Phi R^{\mu} \Phi^{\dagger}\right),
\end{aligned}
$$

where $D^{\mu} \Phi=\partial^{\mu} \Phi-i g_{1}\left(L^{\mu} \Phi-\Phi R^{\mu}\right)$ is the covariant derivative and

$$
\mathcal{L}_{d i l}=\frac{1}{2}\left(\partial_{\mu} G\right)^{2}-\frac{1}{4} \frac{m_{G}^{2}}{\Lambda^{2}}\left(G^{4} \ln \left|\frac{G}{\Lambda}\right|-\frac{G^{4}}{4}\right)
$$

the dilaton (i.e. the scalar glueball) Lagrangian, see Refs. [10, 11] for details.

Spontaneous breaking of chiral symmetry takes place $\left(m_{0}^{2}<0\right)$. As a consequence, one has to perform the shift of the scalar-isoscalar fields by their vacuum expectation values $\phi_{N}$ and $\phi_{S}$ :

$$
\sigma_{N} \rightarrow \sigma_{N}+\phi_{N} \text { and } \sigma_{S} \rightarrow \sigma_{S}+\phi_{S}
$$

In matrix form:

$$
S \rightarrow \Phi_{0}+S \text { with } \Phi_{0}=\frac{1}{\sqrt{2}}\left(\begin{array}{ccc}
\frac{\phi_{N}}{\sqrt{2}} & 0 & 0 \\
0 & \frac{\phi_{N}}{\sqrt{2}} & 0 \\
0 & 0 & \phi_{S}
\end{array}\right) .
$$

In addition, one has also to 'shift' the axial-vector fields

$$
\begin{gathered}
\vec{a}_{1}^{\mu} \rightarrow \vec{a}_{1}^{\mu}+Z_{\pi} w_{\pi} \partial^{\mu} \vec{\pi}, K_{1, A}^{+, \mu} \rightarrow K_{1, A}^{+, \mu}+Z_{K} w_{k} \partial^{\mu} K, \ldots \\
f_{1, N}^{\mu} \rightarrow f_{1, N}^{\mu}+Z_{\eta_{N}} w_{\eta_{N}} \partial^{\mu} \eta_{N}, f_{1, S}^{\mu} \rightarrow f_{1, S}^{\mu}+Z_{\eta_{S}} w_{\eta_{S}} \partial^{\mu} \eta_{S},
\end{gathered}
$$

and to consider the wave-function renormalization of the pseudoscalar fields:

$$
\begin{aligned}
\vec{\pi} & \rightarrow Z_{\pi} \vec{\pi}, K^{+} \rightarrow Z_{K} K^{+}, \ldots \\
\eta_{N} & \rightarrow Z_{\eta_{N}} \eta_{N}, \eta_{S} \rightarrow Z_{\eta_{S}} \eta_{S} .
\end{aligned}
$$

The constants entering into the previous expressions are:

$$
Z_{\pi}=Z_{\eta_{N}}=\frac{m_{a_{1}}}{\sqrt{m_{a_{1}}^{2}-g_{1}^{2} \phi_{N}^{2}}}, Z_{K}=\frac{2 m_{K_{1, A}}}{\sqrt{4 m_{K_{1, A}}^{2}-g_{1}^{2}\left(\phi_{N}+\sqrt{2} \phi_{S}\right)^{2}}}, Z_{\eta_{S}}=\frac{m_{f_{1 S}}}{\sqrt{m_{f_{1 S}}^{2}-2 g_{1}^{2} \phi_{S}^{2}}}
$$


and:

$$
w_{\pi}=w_{\eta_{N}}=\frac{g_{1} \phi_{N}}{m_{a_{1}}^{2}}, \quad w_{K}=\frac{g_{1}\left(\phi_{N}+\sqrt{2} \phi_{S}\right)}{2 m_{K_{1}, A}^{2}}, w_{\eta_{S}}=\frac{\sqrt{2} g_{1} \phi_{S}}{m_{f_{1 S}}^{2}} .
$$

The numerical values of the renormalization constants are $Z_{\pi}=1.709, Z_{K}=1.604, Z_{\eta_{S}}=1.539$ [10], while those of the $w$-parameters are: $w_{\pi}=0.683 \mathrm{GeV}^{-1}, w_{K}=0.611 \mathrm{GeV}^{-1}, w_{\eta_{S}}=0.554 \mathrm{GeV}^{-1}$. Moreover, the condensates $\phi_{N}$ and $\phi_{S}$ read

$$
\phi_{N}=Z_{\pi} f_{\pi}=0.158 \mathrm{GeV}, \phi_{S}=\frac{2 Z_{K} f_{K}-\phi_{N}}{\sqrt{2}}=0.138 \mathrm{GeV},
$$

where the standard values $f_{\pi}=0.0922 \mathrm{GeV}$ and $f_{K}=0.110 \mathrm{GeV}$ have been used [6]. The previous expression can be summarized by the matrix replacements

$$
P \rightarrow \mathcal{P}=\frac{1}{\sqrt{2}}\left(\begin{array}{ccc}
\frac{Z_{\pi}}{\sqrt{2}}\left(\eta_{N}+\pi^{0}\right) & Z_{\pi} \pi^{+} & Z_{K} K^{+} \\
Z_{\pi} \pi^{-} & \frac{Z_{\pi}}{\sqrt{2}}\left(\eta_{N}-\pi^{0}\right) & Z_{K} K^{0} \\
Z_{K} K^{-} & Z_{K} \bar{K}^{0} & Z_{\eta_{S}} \eta_{S}
\end{array}\right)
$$

and

$$
A^{\mu} \rightarrow \mathcal{A}^{\mu}=\frac{1}{\sqrt{2}}\left(\begin{array}{ccc}
\frac{f_{1 N}+a_{1}^{0}}{\sqrt{2}} & a_{1}^{+} & K_{1, A}^{+} \\
a_{1}^{-} & \frac{f_{1 N}-a_{1}^{0}}{\sqrt{2}} & K_{1, A}^{0} \\
K_{1, A}^{-} & \bar{K}_{1, A}^{0} & f_{1 S}
\end{array}\right)^{\mu}+\frac{\partial^{\mu}}{\sqrt{2}}\left(\begin{array}{ccc}
\frac{Z_{\pi} w_{\pi}}{\sqrt{2}}\left(\eta_{N}+\pi^{0}\right) & Z_{\pi} w_{\pi} \pi^{+} & Z_{K} w_{K} K^{+} \\
Z_{\pi} w_{\pi} \pi^{-} & \frac{Z_{\pi} w_{\pi}}{\sqrt{2}}\left(\eta_{N}-\pi^{0}\right) & Z_{K} w_{K} K^{0} \\
Z_{K} w_{K} K^{-} & Z_{K} w_{K} \bar{K}^{0} & Z_{\eta_{S}} w_{\eta_{S}} \eta_{S}
\end{array}\right)
$$

In the $U_{V}(3)$ limit (in which all three bare quark masses are equals), one has: $\Phi_{N}=\sqrt{2} \Phi_{S}, Z=Z_{\pi}=Z_{K}=Z_{\eta_{S}}$, and $w=w_{\pi}=w_{K}=w_{\eta_{S}}$. Hence, in this limit: $P \rightarrow \mathcal{P}=Z P$ and $A^{\mu} \rightarrow \mathcal{A}^{\mu}=A+Z w \partial^{\mu} P$.

The eLSM has been also enlarged to four flavors in Ref. [34]. Interestingly, charmed meson masses and large- $N_{c}$ dominant decays can be described relatively well (even if one is far from the natural domain of chiral symmetry).

In the end, we also recall that the pseudoscalar glueball can be coupled to the eLSM via the chiral Lagrangian $\mathcal{L}_{\tilde{G}}=i c_{\tilde{G} \Phi} \tilde{G}\left(\operatorname{det} \Phi-\operatorname{det} \Phi^{\dagger}\right)$, which reflects the axial anomaly in the pseudoscalar-isoscalar sector, see details and results in Ref. [17, 18]. In a recent extension, the very same Lagrangian is used to study the decay of an hypothetical excited pseudoscalar glueball [21].

\section{Appendix B: Expressions for two-body decays}

The decay $\mathcal{O} \rightarrow b_{1} \pi$ from $\mathcal{L}_{1}$ reads:

$$
\Gamma_{\mathcal{O} \rightarrow b_{1} \pi}=c_{\mathcal{O} b_{1} \pi} \frac{k_{\mathcal{O} b_{1} \pi}}{8 \pi M_{\mathcal{O}}^{2}}\left(\lambda_{1} G_{0} Z_{\pi}\right)^{2} \frac{1}{3}\left(2+\frac{\left(M_{\mathcal{O}}^{2}-m_{\pi}^{2}+m_{b_{1}}^{2}\right)^{2}}{4 M_{\mathcal{O}}^{2} m_{b_{1}}^{2}}\right),
$$

where $c_{\mathcal{O} b_{1} \pi}=3$ is an isospin factor, $M_{\mathcal{O}}=3.8 \mathrm{GeV}$ is the glueball mass, and $m_{\pi}$ and $m_{b_{1}}$ are the pion and $b_{1}$ masses. The quantity $k_{\mathcal{O} b_{1} \pi}$ is the modulus of the three-momentum of one of the two outgoing particles:

$$
k_{\mathcal{O} b_{1} \pi}=\frac{\sqrt{M_{\mathcal{O}}^{4}-2 M_{\mathcal{O}}^{2}\left(m_{\pi}^{2}+m_{b_{1}}^{2}\right)+\left(m_{\pi}^{2}-m_{b_{1}}^{2}\right)^{2}}}{2 M_{\mathcal{O}}} .
$$

The decays of the other channels in Tab. III are calculated in an analogous way, upon taking into account the change of masses, isospin factors, as well as the constants entering in the amplitudes. The two-body decays of $\mathcal{L}_{2}$ presented in Table $\mathrm{V}$ are calculated by using the same procedure.

We now turn to $\mathcal{L}_{3}$. The decay $\mathcal{O} \rightarrow \rho \pi$ reads:

$$
\Gamma_{\mathcal{O} \rightarrow \rho \pi}=c_{\mathcal{O} \rho \pi} \frac{k_{\mathcal{O} \rho \pi}}{8 \pi M_{\mathcal{O}}^{2}}\left(\frac{\alpha}{4} w_{\pi} Z_{\pi} \Phi_{N}^{2}\right)^{2}\left(\frac{2}{3} k_{\mathcal{O} \rho \pi}^{2} M_{\mathcal{O}}^{2}\right)
$$

where $c_{\mathcal{O} \rho \pi}=3$ and $k_{\mathcal{O} \rho \pi}$ is the modulus of the momentum in this case. The other decays $\mathcal{O} \rightarrow V P$ are calculated in the same way. The last decay that we consider is $\mathcal{O} \rightarrow \rho a_{1}(1230)$ :

$$
\Gamma_{\mathcal{O} \rightarrow \rho a_{1}(1230)}=c_{\mathcal{O} \rho a_{1}} \frac{k_{\mathcal{O} \rho a_{1}}}{8 \pi M_{\mathcal{O}}^{2}}\left(\frac{\alpha}{4} \phi_{N}^{2}\right)^{2} \frac{1}{3}\left(6 M_{\mathcal{O}}^{2}+\frac{2 k_{\mathcal{O} \rho a_{1}}^{2} M_{\mathcal{O}}^{2}}{m_{\rho}^{2}}+\frac{2 k_{\mathcal{O} \rho a_{1}}^{2} M_{\mathcal{O}}^{2}}{m_{a_{1}}^{2}}\right)
$$


where $c_{\mathcal{O} \rho a_{1}}=3$ and $k_{\mathcal{O} \rho a_{1}}$ is the corresponding momentum. Analogous decays in Table VI are calculated in a similar way.

\section{Appendix C: Three-body decays of $O$ into two pseudoscalar mesons and a vector meson}

For completeness we report the explicit expression for the three-body decay width of the process $\mathcal{O} \rightarrow P_{1} P_{2} V:$

$$
\Gamma_{\mathcal{O} \rightarrow P_{1} P_{2} V}=\frac{s_{\mathcal{O} \rightarrow P_{1} P_{2} V}}{32(2 \pi)^{3} M_{\mathcal{O}}^{3}} \int_{\left(m_{1}+m_{2}\right)^{2}}^{\left(M_{\mathcal{O}}-m_{3}\right)^{2}} d m_{12}^{2} \int_{\left(m_{23}\right)_{\min }}^{\left(m_{23}\right)_{\max }}\left|-i \mathcal{M}_{\mathcal{O} \rightarrow P_{1} P_{2} V}\right|^{2} d m_{23}^{2}
$$

where (see [6] ):

$$
\begin{aligned}
& \left(m_{23}\right)_{\min }=\left(E_{2}^{*}+E_{3}^{*}\right)^{2}-\left(\sqrt{E_{2}^{* 2}-m_{2}^{2}}+\sqrt{E_{3}^{* 2}-m_{3}^{2}}\right)^{2} \\
& \left(m_{23}\right)_{\max }=\left(E_{2}^{*}+E_{3}^{*}\right)^{2}-\left(\sqrt{E_{2}^{* 2}-m_{2}^{2}}-\sqrt{E_{3}^{* 2}-m_{3}^{2}}\right)^{2}
\end{aligned}
$$

and

$$
E_{2}^{*}=\frac{m_{12}^{2}-m_{1}^{2}+m_{2}^{2}}{2 m_{12}}, E_{3}^{*}=\frac{M_{\mathcal{O}}^{2}-m_{12}^{2}-m_{3}^{2}}{2 m_{12}} .
$$

The quantities $m_{1}$ and $m_{2}$ refer to the masses of the two pseudoscalar states $P_{1}$ and $P_{2}$, while $m_{3}$ refer to the of an (axial-) vector state $V$. We recall also that $m_{i j}^{2}=\left(k_{i}+k_{j}\right)^{2}$ with $k_{1}, k_{2}$, and $k_{3}$ being the four-momenta of the three outgoing particles. Clearly, $p=k_{1}+k_{2}+k_{3}$, where $p$ is the four-momentum of the vector glueball.

The amplitude $\mathcal{M}_{\mathcal{O} \rightarrow P_{1} P_{2} V}$ is calculated at tree-level and $s_{\tilde{G} \rightarrow P_{1} P_{2} V}$ is a symmetrization factor (it equals 1 if $P_{1}$ and $P_{2}$ are different, it equals 2 for $P_{1}=P_{2}$ ). As an example, we consider the decay into $\pi^{0} \pi^{0} \omega$. The amplitude is:

$$
\left|-i \mathcal{M}_{\mathcal{O} \rightarrow \pi^{0} \pi^{0} \omega}\right|^{2}=\frac{\lambda_{2}^{2} Z_{\pi}^{4}}{4} \frac{1}{3}\left(2+\frac{\left(M^{2}+m_{3}^{2}-m_{12}^{2}\right)^{2}}{4 M_{\mathcal{O}}^{2} m_{3}^{2}}\right)
$$

and the symmetry factor is $s_{\mathcal{O} \rightarrow \pi^{0} \pi^{0} \omega}=2$.

[1] F. E. Close, Rept. Prog. Phys. 51, 833 (1988); S. Godfrey and J. Napolitano, Rev. Mod. Phys. 71, 1411 (1999) arXiv:hep-ph/9811410; C. Amsler and N. A. Tornqvist, Phys. Rept. 389, 61 (2004); E. Klempt and A. Zaitsev, Phys. Rept. 454, 1 (2007) arXiv:0708.4016 [hep-ph]].

[2] W. Ochs, J. Phys. G 40 (2013) 043001 arXiv:1301.5183[hep-ph]]. V. Mathieu, N. Kochelev and V. Vento, Int. J. Mod. Phys. E 18 (2009) 1 arXiv:0810.4453 [hep-ph]]. V. Crede and C. A. Meyer, Prog. Part. Nucl. Phys. 63 (2009) 74 [arXiv:0812.0600 [hep-ex]].

[3] Y. Chen, A. Alexandru, S. J. Dong, T. Draper, I. Horvath, F. X. Lee, K. F. Liu and N. Mathur et al., Phys. Rev. D 73, 014516 (2006) arXiv:hep-lat/0510074.

[4] C. Morningstar and M. J. Peardon, AIP Conf. Proc. 688, 220 (2004) arXiv:nucl-th/0309068; C. J. Morningstar and M. J. Peardon, Phys. Rev. D 60 (1999) 034509 hep-lat/9901004]; M. Loan, X. Q. Luo and Z. H. Luo, Int. J. Mod. Phys. A 21, 2905 (2006) arXiv:hep-lat/0503038; E. B. Gregory, A. C. Irving, C. C. McNeile, S. Miller and Z. Sroczynski, PoS LAT2005, 027 (2006) arXiv:hep-lat/0510066.

[5] E. Gregory, A. Irving, B. Lucini, C. McNeile, A. Rago, C. Richards and E. Rinaldi, JHEP 1210 (2012) 170 arXiv:1208.1858 [hep-lat]].

[6] C. Patrignani et al. (Particle Data group), Chin. Phys. C, 40, 100001 (2016).

[7] C. Amsler and F. E. Close, Phys. Rev. D 53 (1996) 295 arXiv:hep-ph/9507326]; M. Strohmeier-Presicek, T. Gutsche, R. Vinh Mau and A. Faessler, Phys. Rev. D 60, 054010 (1999) arXiv:hep-ph/9904461]; W. J. Lee and D. Weingarten, Phys. Rev. D 61, 014015 (2000) arXiv:hep-lat/9910008; F. E. Close and A. Kirk, Eur. Phys. J. C 21, 531 (2001). arXiv:hep-ph/0103173; F. Giacosa, T. Gutsche, V. E. Lyubovitskij and A. Faessler, Phys. Lett. B 622, 277 (2005). arXiv:hep-ph/0504033 ; F. Giacosa, T. Gutsche, V. E. Lyubovitskij and A. Faessler, Phys. Rev. D 72, 094006 (2005) arXiv:hep-ph/0509247. H. Y. Cheng, C. K. Chua and K. F. Liu, Phys. Rev. D 74 (2006) 094005 arXiv:hep-ph/0607206.

[8] A. A. Migdal and M. A. Shifman, Phys. Lett. B 114 (1982) 445. 
[9] A. Salomone, J. Schechter and T. Tudron, Phys. Rev. D 23, 1143 (1981) H. Gomm and J. Schechter, Phys. Lett. B 158, 449 (1985); R. Gomm, P. Jain, R. Johnson and J. Schechter, Phys. Rev. D 33, 801 (1986); J. Schechter, AIP Conf. Proc. 1361 (2011) 60.

[10] D. Parganlija, P. Kovacs, G. Wolf, F. Giacosa and D. H. Rischke, Phys. Rev. D 87 (2013) 014011 [arXiv:1208.0585][hep-ph]].

[11] S. Janowski, F. Giacosa and D. H. Rischke, arXiv:1408.4921 [hep-ph].

[12] L. -C. Gui, Y. Chen, G. Li, C. Liu, Y. -B. Liu, J. -P. Ma, Y. -B. Yang and J. -B. Zhang, Phys. Rev. Lett. 110 (2013) 021601 arXiv:1206.0125 [hep-lat]].

[13] F. Brünner, D. Parganlija and A. Rebhan, Phys. Rev. D 91 (2015) 10, 106002 arXiv:1501.07906 [hep-ph]]; F. Brünner and A. Rebhan, Phys. Rev. Lett. 115 (2015) no.13, 131601 arXiv:1504.05815 [hep-ph]]; F. Brünner and A. Rebhan, Phys. Rev. D 92 (2015) no.12, 121902 arXiv:1510.07605 [hep-ph]].

[14] A. Masoni, C. Cicalo and G. L. Usai, J. Phys. G 32 (2006) R293.

[15] C. Rosenzweig, A. Salomone and J. Schechter, Phys. Rev. D 24 (1981) 2545; C. Rosenzweig, J. Schechter and C. G. Trahern, Phys. Rev. D 21 (1980) 3388; C. Rosenzweig, A. Salomone and J. Schechter, Nucl. Phys. B 206 (1982) 12 [Erratum-ibid. B 207 (1982) 546]; K. Kawarabayashi and N. Ohta, Nucl. Phys. B 175 (1980) 477.

[16] T. Gutsche, V. E. Lyubovitskij and M. C. Tichy, Phys. Rev. D 80 (2009) 014014 arXiv:0904.3414 [hep-ph]].

[17] W. I. Eshraim, S. Janowski, F. Giacosa and D. H. Rischke, Phys. Rev. D 87 (2013) 5, 054036 arXiv:1208.6474 [hep-ph]].

[18] W. I. Eshraim, S. Janowski, A. Peters, K. Neuschwander and F. Giacosa, Acta Phys. Polon. Supp. 5 (2012) 1101 arXiv:1209.3976 [hep-ph]].

[19] M. F. M. Lutz et al. [ PANDA Collaboration ], arXiv:0903.3905 [hep-ex]].

[20] M. Ablikim et al. (BES Collaboration), Phys. Rev. Lett. 95, 262001 (2005); N. Kochelev and D. P. Min, Phys. Lett. B 633, 283 (2006); M. Ablikim et al. (BES III Collaboration), Phys. Rev. Lett. 106.072002 (2011).

[21] W. I. Eshraim and S. Schramm, Phys. Rev. D 95 (2017) no.1, 014028 [arXiv:1606.02207 [hep-ph]].

[22] J. Z. Bai et al. [BES Collaboration], Phys. Rev. D 54 (1996) 1221 Erratum: [Phys. Rev. D 57 (1998) 3187 ].

[23] D. Robson, doi:10.1016/0370-2693(77)90877-2

[24] G. W. S. Hou, In *Minneapolis 1996, Particles and fields, vol. 1* 399-401 hep-ph/9609363. G. W. S. Hou, hep-ph/9707526 C. T. Chan and W. S. Hou, Nucl. Phys. A 675 (2000) 367C hep-ph/9911423.

[25] M. Suzuki, Phys. Rev. D 65 (2002) 097507 hep-ph/0203012.

[26] S. J. Brodsky, G. P. Lepage and S. F. Tuan, Phys. Rev. Lett. 59 (1987) 621.

[27] G. Amelino-Camelia et al., Eur. Phys. J. C 68 (2010) 619 arXiv:1003.3868 [hep-ex]].

[28] R. L. Jaffe, Phys. Rev. D 15 (1977) 267. R. L. Jaffe, Phys. Rev. D 15 (1977) 281.

[29] L. Maiani, F. Piccinini, A. D. Polosa and V. Riquer, Phys. Rev. Lett. 93 (2004) 212002 arXiv:hep-ph/0407017; F. Giacosa, Phys. Rev. D 74 (2006) 014028 arXiv:hep-ph/0605191]; A. H. Fariborz, R. Jora and J. Schechter, Phys. Rev. D 72 (2005) 034001 arXiv:hep-ph/0506170; A. H. Fariborz, Int. J. Mod. Phys. A 19 (2004) 2095; arXiv:hep-ph/0302133. M. Napsuciale and S. Rodriguez, Phys. Rev. D 70 (2004) 094043. F. Giacosa, Phys. Rev. D 75 (2007) 054007 arXiv:hep-ph/0611388.

[30] E. van Beveren, T. A. Rijken, K. Metzger, C. Dullemond, G. Rupp and J. E. Ribeiro, Z. Phys. C 30, 615 (1986) arXiv:0710.4067 [hep-ph]]. E. van Beveren, D. V. Bugg, F. Kleefeld and G. Rupp, Phys. Lett. B 641, 265 (2006) arXiv:hep-ph/0606022. J. R. Pelaez, Phys. Rev. Lett. 92, 102001 (2004) arXiv:hep-ph/0309292; J. A. Oller and E. Oset, Nucl. Phys. A 620, 438 (1997) [Erratum-ibid. A 652, 407 (1999)] arXiv:hep-ph/9702314]. J. A. Oller, E. Oset and J. R. Pelaez, Phys. Rev. D 59 (1999) 074001 [Erratum-ibid. D 60 (1999 ERRAT,D75,099903.2007) 099906] arXiv:hep-ph/9804209.

[31] J. R. Pelaez, arXiv:1510.00653 [hep-ph].

[32] N. A. Törnqvist, Z. Phys. C68, 647-660 (1995) arXiv:hep-ph/9504372; N. A. Törnqvist and M. Roos, Phys. Rev. Lett. 76, 1575-1578 (1996) arXiv:hep-ph/9511210v1]; M. Boglione and M. R. Pennington, Phys. Rev. Lett. 79, 1998-2001 (1997) arXiv:hep-ph/9703257; M. Boglione and M. R. Pennington, Phys. Rev. D65, 114010 (2002) arXiv:hep-ph/0203149; T. Wolkanowski, F. Giacosa and D. H. Rischke, Phys. Rev. D 93 (2016) no.1, 014002 arXiv:1508.00372 [hep-ph]]; T. Wolkanowski, M. Soltysiak and F. Giacosa, Nucl. Phys. B 909 (2016) 418 arXiv:1512.01071 [hep-ph]].

[33] D. Parganlija, F. Giacosa and D. H. Rischke, Phys. Rev. D 82, 054024 (2010) arXiv:1003.4934 [hep-ph]]; S. Janowski, D. Parganlija, F. Giacosa, D. H. Rischke, Phys. Rev. D84 (2011) 054007. arXiv:1103.3238 [hep-ph]].

[34] W. I. Eshraim, F. Giacosa and D. H. Rischke, Eur. Phys. J. A 51 (2015) 9, 112 arXiv:1405.5861 [hep-ph]].

[35] S. Gallas, F. Giacosa and D. H. Rischke, Phys. Rev. D 82 (2010) 014004 arXiv:0907.5084 [hep-ph]]; L. Olbrich, M. Zétényi, F. Giacosa and D. H. Rischke, Phys. Rev. D 93 (2016) no.3, 034021 arXiv:1511.05035 [hep-ph]].

[36] S. Gallas, F. Giacosa and G. Pagliara, Nucl. Phys. A 872 (2011) 13 arXiv:1105.5003 [hep-ph]]; A. Heinz, F. Giacosa and D. H. Rischke, Nucl. Phys. A 933 (2015) 34 [arXiv:1312.3244 [nucl-th]].

[37] P. Kovács, Z. Szép and G. Wolf, Phys. Rev. D 93 (2016) no.11, 114014 arXiv:1601.05291 [hep-ph]]; A. N. Tawfik and A. M. Diab, Phys. Rev. C 91 (2015) no.1, 015204 arXiv:1412.2395 [hep-ph]].

[38] F. Divotgey, P. Kovacs, F. Giacosa and D. H. Rischke, arXiv:1605.05154 [hep-ph].

[39] F. Divotgey, L. Olbrich and F. Giacosa, Eur. Phys. J. A 49 (2013) 135 arXiv:1306.1193 [hep-ph]].

[40] F. Giacosa, T. Gutsche, V. E. Lyubovitskij and A. Faessler, Phys. Rev. D 72 (2005) 114021 arXiv:hep-ph/0511171.

[41] L. Burakovsky and J. T. Goldman, Phys. Rev. D 57, 2879 (1998) hep-ph/9703271.

[42] R. Escribano, Eur. Phys. J. C 65 (2010) 467 arXiv:0807.4201 [hep-ph]].

[43] F. E. Close and Q. Zhao, Phys. Rev. D 71 (2005) 094022 hep-ph/0504043.

[44] S. Strauss, C. S. Fischer and C. Kellermann, Phys. Rev. Lett. 109 (2012) 252001 arXiv:1208.6239 [hep-ph]]. A. C. Aguilar, D. Binosi and J. Papavassiliou, Phys. Rev. D 84 (2011) 085026 [arXiv:1107.3968 [hep-ph]]. A. C. Aguilar and A. A. Natale, JHEP 0408 (2004) 057 hep-ph/0408254. 
[45] M. Loan and Y. Ying, Prog. Theor. Phys. 116 (2006) 169 hep-lat/0603030.

[46] W. Sun et al., arXiv:1702.08174 [hep-lat].

[47] J. J. Dudek, R. G. Edwards, B. Joo, M. J. Peardon, D. G. Richards and C. E. Thomas, Phys. Rev. D 83 (2011) 111502 arXiv:1102.4299 [hep-lat]].

[48] J. J. Dudek et al. [Hadron Spectrum Collaboration], Phys. Rev. D 88 (2013) no.9, 094505 arXiv:1309.2608 [hep-lat]].

[49] F. Giacosa, EPJ Web Conf. 130 (2016) 01009 arXiv:1609.08055 [hep-ph]].

[50] R. L. Jaffe, K. Johnson and Z. Ryzak, Annals Phys. 168 (1986) 344.

[51] R. C. Brower, S. D. Mathur and C. I. Tan, Nucl. Phys. B 587 (2000) 249 hep-th/0003115.

[52] M. Boglione and M. R. Pennington, Phys. Rev. Lett. 79 (1997) 1998 hep-ph/9703257. F. Giacosa and G. Pagliara, Phys. Rev. C 76 (2007) 065204 arXiv:0707.3594 [hep-ph]].

[53] G. 't Hooft, Nucl. Phys. B 72 (1974) 461.

[54] E. Witten, Nucl. Phys. B 160 (1979) 57.

[55] S. R. Coleman, Lectures given at 17th International School of Subnuclear Physics: Pointlike Structures Inside and Outside Hadrons 31 Jul - 10 Aug 1979. Erice, Italy. SLAC-PUB-2484.

[56] R. F. Lebed, Czech. J. Phys. 49 (1999) 1273 nucl-th/9810080.

[57] S. Okubo, Phys. Lett. 5, 165 (1963). G. Zweig, CERN Report No.8419/TH412 (1964). J. Iizuka, Prog. Theor. Phys. Suppl. 37 (1966) 21.

[58] B. R. Martin and G. Shaw, "Particle physics," Chichester, UK: Wiley (2008) 442 p. 